\title{
Diseño y Construcción de Equipo para Realizar Prospección Geofísica Aplicando el Método VDE Tomografía ELÉctrica
}

\author{
Design and Construction of Equipment for Applying the \\ Geophysical Prospecting Method Electric Tomography
}

\author{
Giraldo Sánchez Héctor Fabio', Sendoya Losada Diego Fernando² \\ ${ }^{1}$ Escuela de Ciencias Básicas, Tecnología e Ingeniería, Universidad Nacional Abierta y a Distancia-UNAD, \\ Neiva, Colombia, hfgiraldos@hotmail.com \\ 2Escuela de Ciencias Básicas, Tecnología e Ingeniería, Universidad Nacional Abierta y a Distancia-UNAD, \\ Neiva, Colombia, diego.sendoya@unad.edu.co.
}

Recibido: 12/08/2012・ Aprobado.15/11/2012

\section{RESUMEN}

En este artículo, se expone el procedimiento del diseño y la construcción de un equipo eléctrico para realizar prospección geofísica por medio del método de tomografía eléctrica. El equipo es de media potencia, lo que garantiza profundidades de exploración bastante adecuadas para aplicaciones y estudios comerciales y de geotecnia. Así mismo, es en esencia, una fuente de voltaje DC de 500 voltios que está en capacidad de proporcionar una intensidad de corriente máxima de 1 Amperio. Consta además de una pequeña fuente de corriente encargada de contrarrestar las corrientes eléctricas que de forma natural se hallan en el subsuelo y que se manifiestan como una diferencia de potencial en la superficie. Una explicación general del método geofísico en mención, permite entender los principios básicos de funcionamiento del equipo y las funciones que ha de cumplir.

Una vez finalizada la construcción del equipo, se recolectaron algunos datos en campo, en zona cercana al municipio de Gachancipá, Cundinamarca. Estos datos se procesaron con un software especializado.

Por otra parte, las imágenes obtenidas con el software presentaron las distribuciones de las resistividades del subsuelo que se pueden asociar a las posibles estructuras y geología del área de estudio.

Palabras clave: geoeléctrica, geofísica, resistividad aparente, prospección geofísica, sondeo eléctrico vertical (SEV), tomografía eléctrica 


\section{Abstract}

Outlines the procedure for the design and construction of electric equipment for geophysical prospecting through electrical tomography method. The team is of average power, ensuring exploration depths quite suitable for applications and commercial and geotechnical studies. The device is essentially a DC voltage source of 500 volts that is able to provide a maximum current of $1 \mathrm{amp}$. It also contains a small charge current source of electrical currents counteract naturally found in the subsoil and are manifested as a difference in the surface potential. A general explanation of the geophysical method in question, helps to understand the basic principles of operation of the equipment and functions to be fulfilled.

After building the team, we conducted a field data acquisition, in area near the town of Gachancipa Cundinamarca. The data from this equipment are processed with specialized software. The images obtained with the software presents the distributions of subsurface resistivity can be associated with the possible structures and geology of the study area.

Keywords: apparent resistivity, electrical tomography, geoelectric, geophysics, geophysical prospecting, vertical electrical sounding (VES)

\section{INTRODUCCIÓN}

El estudio del comportamiento eléctrico de las rocas y los sedimentos en relación con la corriente eléctrica se denomina geoeléctrica. La geoeléctrica es un método geofísico que permite delimitar las capas del subsuelo obteniendo de éste espesores y resistividades eléctricas de capas características.

Los métodos geoeléctricos consisten en introducir una corriente eléctrica en el suelo y medir caídas de tensión producidas por esta, en distintos puntos de la superficie. Este procedimiento permite obtener valores de resistividades aparentes para diferenciar zonas del subsuelo a partir de valores de corriente y caída de tensión. Cada tipo de material o estructura en el subsuelo presenta un rango de resistividades características, cuyo valor sirve de base para la interpretación de los resultados.

Las investigaciones de resistividad dan una imagen de la distribución de resistividad del subsuelo. Para convertir esa imagen en una estructura geológica, es muy importante conocer cómo varía este parámetro en los diferentes tipos de materiales que constituyen el subsuelo y en las estructuras geológicas del área bajo estudio.

Se diseña e implementa un equipo eléctrico destinado a servir como herramienta de adquisición de datos geofísicos de resistividad eléctrica, aplicando la técnica de tomografía eléctrica. Se concibe un equipo de aproximadamente 500W de potencia, ya que con esta capacidad se pueden realizar estudios generales de interés geológico y geotécnico [1].

El equipo permite realizar investigaciones en el subsuelo a profundidades no mayores a 300 metros (aunque la profundidad efectiva de investigación depende de las características locales del subsuelo) y obtener medidas de voltaje y de corriente, con las cuales se pueda calcular la resistividad aparente en distintos puntos del subsuelo.

Para lograr una imagen tomográfica del subsuelo es necesario alimentar un software de inversión especializado con los datos obtenidos con el equipo. El equipo para realizar tomografía 
eléctrica permite hacer estudios cuyos resultados son de particular interés en hidrogeología, ya que se logra determinar niveles freáticos o la presencia de reservorios acuíferos, con la particularidad de poder hacer estos estudios a un bajo costo, comparado con el uso de otros equipos o medios mecánicos (perforaciones).

En los elementos necesarios para la aplicación completa del método geoeléctrico, se cuentan el equipo de adquisición, las herramientas de campo, el software de procesamiento y, finalmente, la interpretación de los resultados obtenidos. En cuanto al equipo de adquisición, existen distintas configuraciones electródicas que puede adoptar el equipo. Con respecto a las partes del equipo, destaca entre ellas la denominada unidad central, objeto central y de interés en este artículo.

\section{Principios del Método Geoeléctrico}

La Fig. 1 ilustra el principio fundamental de la medida de resistividad del subsuelo, el cual consiste en inyectar una corriente eléctrica continua a través de los electrodos $\mathrm{A}$ y $\mathrm{B}$, determinándose la diferencia de potencial que existe entre el par de electrodos $\mathrm{M}$ y $\mathrm{N}$.

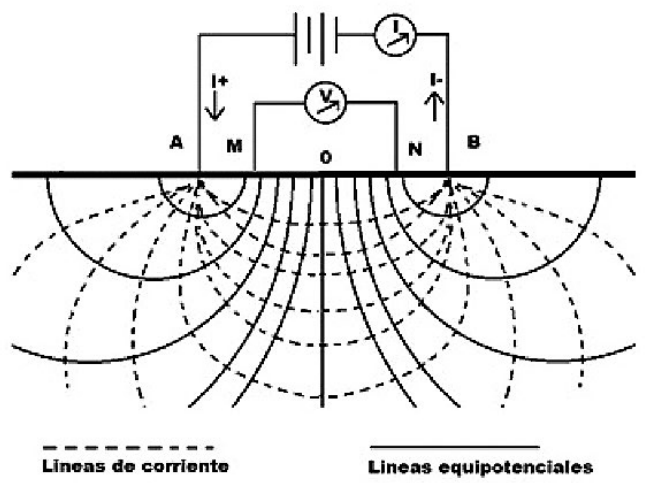

Fig. 1 Esquema básico de dispositivo para determinar la resistividad eléctrica del subsuelo.

Los electrodos de inyección de corriente A y $B$ y los de medición $\mathrm{M}$ y $\mathrm{N}$ se disponen alineados, de acuerdo con las configuraciones propuestas por Schlumberger y Wenner [2].
Para un medio homogéneo de resistividad, la diferencia de potencial es [3].

$$
\begin{array}{r}
\Delta V=\frac{I \rho}{2 \pi}\left(\frac{1}{A M}-\frac{1}{A N}-\frac{1}{B M}+\frac{1}{B N}\right) \\
\Delta V=\frac{I \rho}{2 \pi}\left(\frac{1}{A M}-\frac{1}{A N}-\frac{1}{B M}+\frac{1}{B N}\right)
\end{array}
$$

Donde AM, AN, BM, BN son las distancias entre los electrodos. La resistividad viene dada por la ecuación (2):

$$
\rho=g \frac{\Delta V}{I} \quad \rho=g \frac{\Delta V}{I}
$$

donde:

$$
\begin{gathered}
g=2 \pi\left(\frac{1}{A M}-\frac{1}{A N}-\frac{1}{B M}+\frac{1}{B N}\right)^{-1} \\
g=2 \pi\left(\frac{1}{A M}-\frac{1}{A N}-\frac{1}{B M}+\frac{1}{B N}\right)^{-1}
\end{gathered}
$$

Como se observa en (3) g es un factor geométrico que depende del arreglo de los electrodos.

La técnica de la tomografía eléctrica emplea los valores de resistividad aparente, medidos con los dispositivos geoeléctricos sobre la superficie del terreno, para generar imágenes del subsuelo donde se representan los valores de la resistividad verdadera de las diferentes zonas. La relación entre la resistividad aparente y la resistividad verdadera es compleja. Para determinar la resistividad verdadera del subsuelo a partir de los valores de la resistividad aparente, se aplica la técnica de la "inversión" [4].

\section{Equipo de Adquisición}

El equipo que se diseña y construye está esencialmente conformado por las siguientes partes:

A. Unidad central: es la encargada de proporcionar el voltaje y la corriente que han de ser introducidos en el subsuelo. Cuenta con medidores que proporcionan el valor de las magnitudes 
de la corriente introducida en el subsuelo, y de la diferencia de potencial medida en el mismo.

B. Los electrodos: son barras metálicas que se introducen en el suelo; su longitud debe ser la suficiente como para poderse fijar firmemente en cualquier terreno, ya que su función es la de proporcionar el contacto eléctrico entre el equipo y el suelo.

C. Los cables o conductores de cobre: su longitud depende de la distancia máxima que podría haber entre el equipo y cualquiera de los electrodos; su función es la de conducir la corriente eléctrica desde el equipo hasta los electrodos y/o viceversa.

D. Los conectores: se hallan en los extremos de los cables y pueden ser pinzas del tamaño suficiente para poder ser agarradas a los electrodos o conectores adecuados con el fin de lograr una conexión firme entre los cables y el equipo.

E. Batería: es la fuente de alimentación que proporciona energía a todo el sistema; debe cumplir con la condición de ser portable, recargable y fácil de reemplazar

\section{Generalidades de la Unidad Central}

La unidad central del equipo de adquisición se puede dividir en dos circuitos generales: uno de emisión y otro de recepción.

\section{A. Circuito de emisión.}

Su finalidad es hacer circular una corriente eléctrica de intensidad constante I por el suelo, introduciéndola a través de dos electrodos A y B. Este circuito está conformado por una fuente de alimentación, una etapa de adecuación de corriente y voltaje, dos electrodos $\mathrm{A}$ y $\mathrm{B}$, un amperímetro o miliamperímetro para la medición de I, los cables y elementos de conexión necesarios y finalmente el suelo como carga eléctrica.
El equipo está en capacidad de entregar una diferencia de tensión máxima de 500 voltios DC en sus terminales de salida A y B; para ello, el circuito emisor toma la tensión de entrada de 12 voltios DC entregada por la fuente de alimentación o batería y la convierte en una tensión alterna. Esta tensión alterna se usa para alimentar un transformador elevador que convierte la señal de entrada de 12 voltios pico a pico, en una señal de salida de 500 voltios AC. Esta señal se rectifica, con lo que se obtiene una salida de 500 voltios DC entre los terminales A y B. En la Fig. 2 se ilustra el diagrama de bloques del circuito emisor.

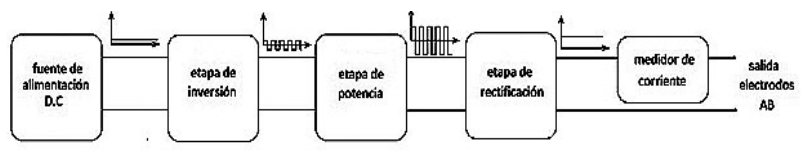

Fig 2. Diagrama de bloques del circuito emisor.

El circuito inversor es en esencia un conversor de potencia que se alimenta de un voltaje DC de 12 voltios y entrega un voltaje de salida de 500 voltios DC a un amperio; es decir, con una potencia de 500 vatios.

En este circuito, el integrado CD4047, el cual es un multivibrador CMOS monoestable/ estable de bajo poder, se utiliza para generar una señal de onda cuadrada de $60 \mathrm{HZ}$. Esta podría considerase una etapa de inversión de la señal de entrada de DC a AC.

Luego de que la señal se invierte, un amplificador operacional de la serie LM358 amplifica cada uno de los pulsos negativo y positivo. EI LM358 contiene dos amplificadores operacionales independientes de alta ganancia, con compensación interna.

La señal de salida de los LM358 se amplifica por transistores NPN de la serie 2N2222, los cuales están en capacidad de disipar una potencia máxima de 25 Watts.

La etapa de potencia está compuesta por dos juegos de cuatro transistores NPN de la serie 
2N3055 cada uno, controlados por la salida de los 2N2222. Estos transistores se han diseñado para usos de amplificación de propósito general y para aplicaciones de switcheo, por lo que son fáciles de adquirir y reemplazar. Cada uno de estos juegos está conectado al devanado central del primario de un transformador elevador que recibirá un voltaje de 12 voltios $A C$ y está en capacidad de entregar un voltaje de salida de 500 voltios AC.

Finalmente, el voltaje, que es entregado por el devanado secundario del transformador, se rectifica y se convierte en un voltaje de unos 500 voltios DC.

\section{B. Circuito de recepción.}

La finalidad de este circuito es suministrar la medida de diferencia de potencial proporcionada por el suelo excitado con el circuito de emisión. Esto se hace a través de los electrodos $\mathrm{M}$ y $\mathrm{N}$ y de un voltímetro que medirá dicha diferencia de potencial, además de cables de conexión, necesarios entre ellos [3].

Adicional a esto, este equipo cuenta con una pequeña fuente de corriente aislada del circuito de emisión, que cumple la función de inyectar una pequeña corriente eléctrica directa al suelo a través de los electrodos $\mathrm{M}$ y $\mathrm{N}$, con el fin de anular las corrientes telúricas que se hallen en la zona de estos electrodos y así lograr eliminar el potencial espontáneo que exista, antes de inyectar alguna corriente, por los electrodos A y B.

En resumen, el circuito receptor es básicamente un voltímetro conectado a los electrodos $\mathrm{M}$ y $\mathrm{N}$, pero, además, en paralelo a este, hay un circuito generador de corriente destinado a compensar los potenciales espontáneos. Para ello cuenta con una fuente de voltaje DC de 6 voltios, la cual es una pequeña batería recargable independiente a la de 12 voltios DC. Esta batería se halla conectada en paralelo a un selector de seis posiciones, el cual se encarga de invertir la polaridad de salida del circuito y de seleccionar resistencias de valores diferentes para ajustar la corriente de salida. Con el fin de proporcionar un ajuste fino para esta corriente se conecta un potenciómetro en serie con la fuente de 6 voltios.

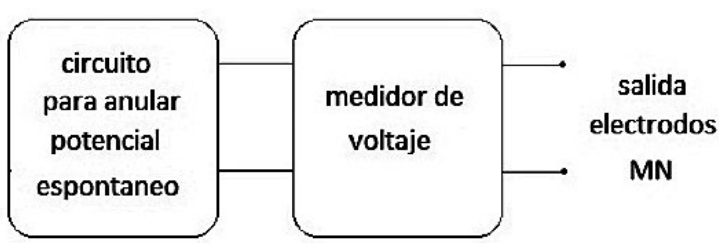

Fig. 3 Diagrama de bloques del circuito receptor.

En la Fig. 4 se puede apreciar el diagrama de bloques del equipo, en donde los bloques superiores corresponden al circuito de emisión, y los bloques inferiores, al circuito de recepción

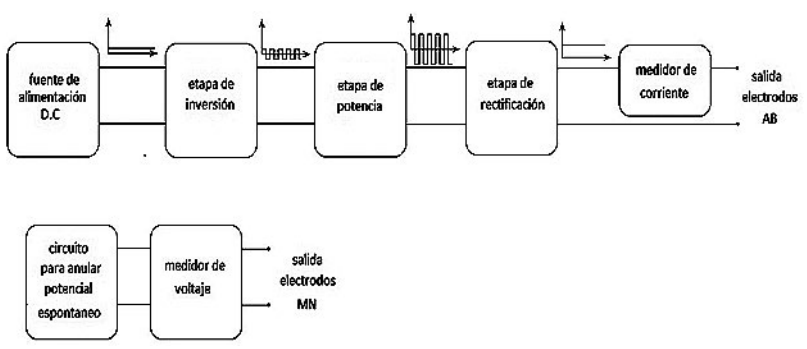

Fig. 4 Diagrama de bloques de la unidad central del equipo de adquisición

\section{Descripción del panel frontal.}

El panel frontal de la unidad central del equipo de adquisición contiene los indicadores y controles del equipo, los interruptores de encendido y apagado, además de los puertos de conexión a los electrodos $\mathrm{A}$ y $\mathrm{B}$, a los electrodos $\mathrm{M}$ y $\mathrm{N}$ y a la fuente de alimentación.

Los indicadores son multímetros digitales de marca UNI-T de referencia UT33C, los cuales son muy fiables y relativamente fáciles de conseguir en el mercado, por lo que en caso de avería se pueden reemplazar rápida y fácilmente. El multímetro ubicado en el borde izquierdo del panel frontal es el encargado de medir la corriente que circula a través de los electrodos A y B. Esto lo hace midiendo la caída de tensión que se genera en una resistencia de $1 \Omega$ la cual se halla en serie con el circuito generador. El segundo multímetro es el encargado de registrar la caída de tensión 
entre los electrodos $\mathrm{M}$ y $\mathrm{N}$ y el valor del potencial espontáneo presente entre los mismos, en un momento determinado.

El panel frontal cuenta con los puertos de salida de los electrodos $\mathrm{M}$ y $\mathrm{N}$, y de los electrodos $\mathrm{A}$ y $B$. Se debe tener mucha precaución con estos últimos, pues cuando el equipo se halla activo, a través de estos electrodos se presenta una diferencia de potencial cercana a los 500 voltios DC con una corriente máxima de un amperio; por lo tanto, existe un riesgo alto de lesiones o descargas eléctricas mortales si es manipulado de manera inadecuada. En la Fig. 5 se observa el panel frontal del equipo.

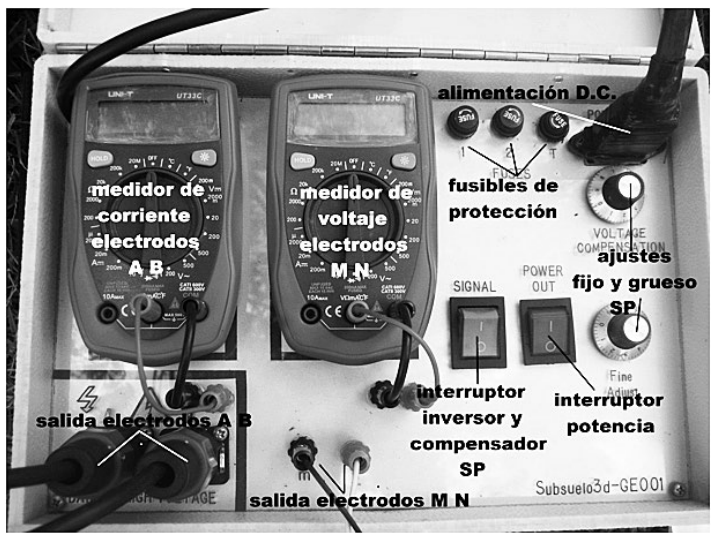

Fig. 5 Panel frontal del equipo.

El panel frontal contiene el conector para la fuente de alimentación, la cual se halla fuera de la unidad central; esto se hace para permitir que el equipo sea más portable, y para que en caso de avería de la fuente de alimentación, esta pueda reemplazarse fácilmente. Son observables también en él, los interruptores de alimentación para los circuitos emisor y el receptor, además de los controles de ajuste fino y grueso para lograr una adecuada anulación del potencial espontáneo.

\section{Fuente de alimentación.}

La fuente de alimentación usada para suministrar energía al sistema es una batería recargable externa de 12 Voltios con capacidad de suministrar una corriente de 50 o 100 amperios-hora. Este tipo de batería se utiliza comúnmente en au- tomóviles, lo que permite intercambiarla fácilmente y contar con una buena cantidad de energía, necesaria en largas jornadas de uso.

Esta fuente de alimentación se conecta a la unidad central por medio de un par de hilos conductores con caimanes y conectores resistentes en sus extremos. Estos conductores están en capacidad de soportar una corriente DC máxima de 40 amperios, corriente, que sería la máxima, en caso de someter el equipo a condiciones de resistividad del terreno muy bajas.

\section{Partes de la Unidad Central}

\section{A. Oscilador.}

El voltaje DC que alimenta la unidad central se convierte en una señal de onda cuadrada con el uso de un integrado CD4047. El circuito integrado CMOS CD4047 es un oscilador astable que tiene tres salidas de frecuencia: la principal está ubicada en la patilla 13 y las otras dos son salidas en forma de flip-flop, una negada y la otra normal; estas dos salidas son divisiones de frecuencia, es decir, que las otras salidas son equivalentes a la mitad de la frecuencia principal. Estas salidas son los terminales 10 y 11 del integrado y son las que se utilizan para el propósito de este trabajo. La ventaja de este oscilador radica en que su ciclo de trabajo es constante y la frecuencia puede variarse con un potenciómetro; el voltaje de entrada puede ser de 15 voltios máximo, con una frecuencia de oscilación máxima de $10 \mathrm{MHz}$.

Para configurar el circuito oscilador se utilizaron los siguientes componentes:

\section{CMOS CD4047.}

1 capacitor electrolítico de $0.1 \mu \mathrm{F}$ a 10 voltios.

1 resistencia variable de $100 \mathrm{~K} \Omega$.

2 resistencias de $4.7 \mathrm{~K} \Omega$ a $1 / 2$ watt.

El circuito oscilador se alimenta con la fuente de alimentación de 12 voltios. En la Fig. 6 se observa el diagrama esquemático del circuito oscilador. 


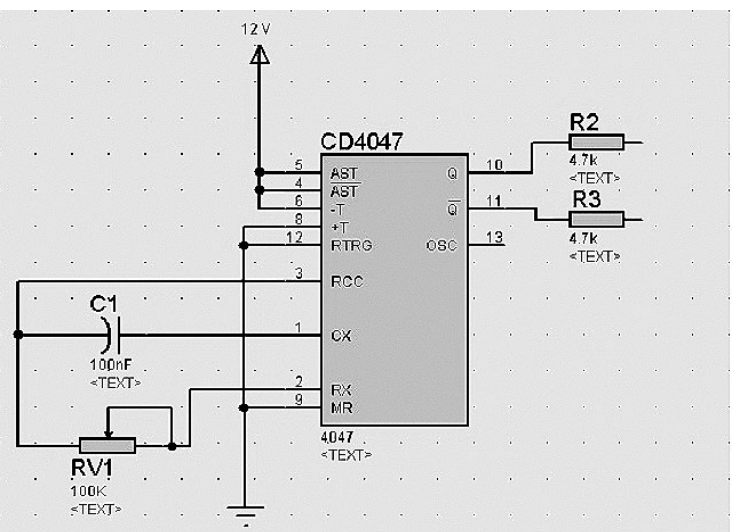

Fig. 6 Circuito oscilador.

\section{B. Preamplificador.}

Las señales de salida de los pines 10 y 11 del CD4047 son las de entrada positiva de los amplificadores operacionales empaquetados en el LM 358. Estos Amp Op están configurados en el modo de realimentación negativa, y sus salidas, los pines 1 y 7, alimentan la base de los 2N2222. El LM358 ,al igual que el CD4047, se alimentan de la fuente de $12 \mathrm{~V} \mathrm{DC}$.

EI LM358 es un gran amplificador operacional de doble canal, fácil de usar. Las aplicaciones del LM358 incluyen amplificadores de transductor, DC bloques de ganancia y todos los circuitos de amplificador operacional convencional. Puede manejar de 3-32VDC y la fuente de hasta $20 \mathrm{~mA}$ por canal. Este amplificador operacional es ideal si se necesita para operar dos amplificadores operacionales individuales de una sola fuente de alimentación. En la Fig. 7 se observa el diagrama esquemático de la etapa de pre amplificación.

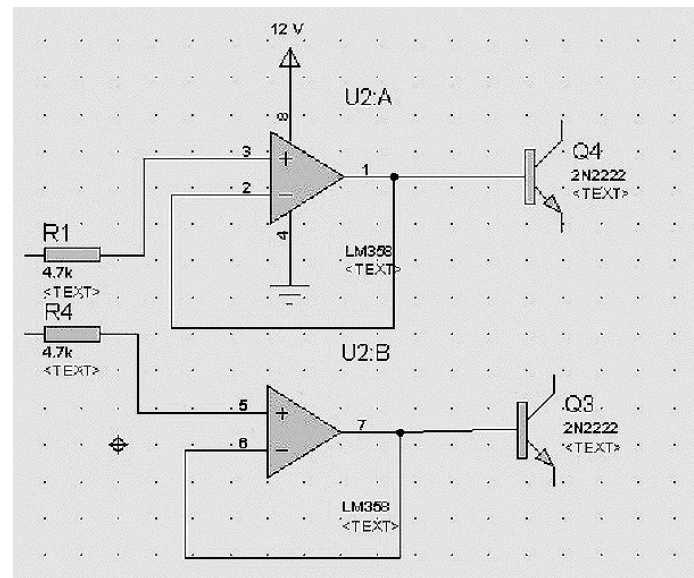

Fig. 7 Preamplificador.

\section{Amplificador.}

El circuito encargado de amplificar la corriente y que le da al equipo la capacidad de suministrar los 500W de potencia está constituido por 10 transistores 2N3055 configurados como sigue: un 2N2222 amplifica la señal que entrega el LM358 en una de sus salidas; cada salida corresponde a un pulso (negativo o positivo) del CD4047. La señal amplificada por el 2N2222 es re amplificada por un 2N3055 que se halla configurado en cascada con el anterior. La señal que entrega este 2N3055 alimenta la base de otros cuatro 2N3055 dispuestos en paralelo. Estos 2N3055 se alimentan de la fuente de $12 \mathrm{~V}$ DC que se conecta al devanado central del primario del transformador Fig. 8.

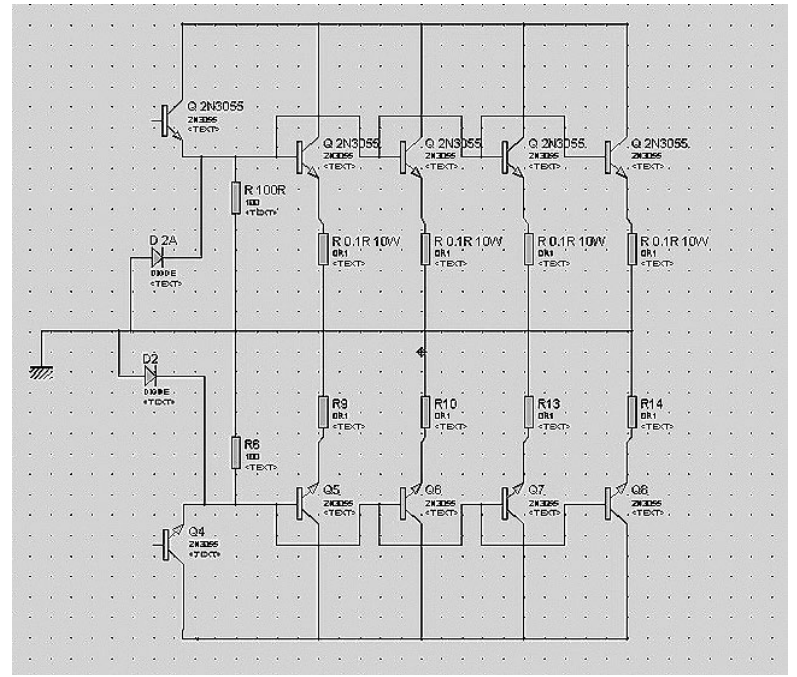

Fig. 8 Etapa de potencia.

\section{Elevador y rectificador.}

La fuente de alimentación de $12 \mathrm{~V}$ DC se conecta al devanado central del primario del transformador y cada una de las otras terminales del primario se conectan a los colectores de los 2N3055, descritos anteriormente. Como cada grupo de 2N3055 conduce corriente alternadamente, esto induce un voltaje en el secundario del transformador. Las especificaciones del transformador son $12 \mathrm{~V}-0-12 \mathrm{~V}$ en el primario y de $500 \mathrm{~V}$ en el secundario. El voltaje de salida en el secundario se rectifica y filtra; para ello, se usa un puente rectificador de 3 amperios y un capacitor de alto voltaje. Para medir la corriente de salida 
del secundario se hace circular esta por una resistencia de $1 \Omega$, lo que permite medir una caida de tensión sobre la misma, igual a la corriente que circula por ella. En la Fig. 9 se aprecia el diagrama esquemático del elevador y el rectificador.

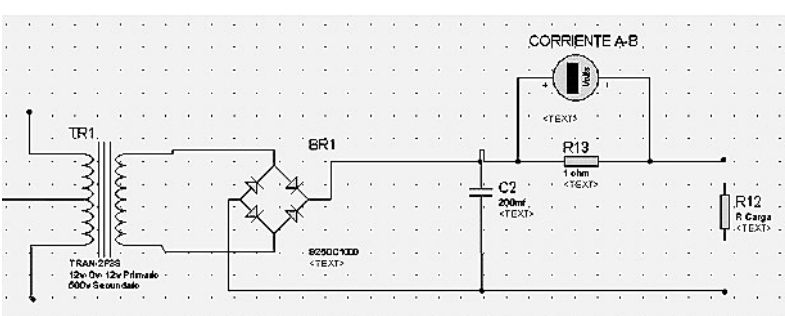

Fig. 9 Transformador elevador y circuito rectificador.

\section{E. Compensador.}

El circuito compensador es una pequeña fuente alimentada por una batería de $6 \mathrm{~V}$ DC. Para regular la corriente de salida del circuito, este está configurado con un selector de seis posiciones y un potenciómetro, lo que permite variar el valor de la resistencia del circuito. Este circuito está conectado en paralelo a un voltímetro, el cual se encarga de verificar que se compensen correctamente los potenciales espontáneos y de medir la diferencia de potencial entre los electrodos $\mathrm{M}$ y $\mathrm{N}$ cuando se excitan los electrodos A y B.

\section{Resultados}

Una vez se construyó el equipo de adquisición, se utilizó para obtener una serie de datos geoeléctricos en la zona norte del municipio de Gachancipá, Cundinamarca. La línea de adquisición se planteó cerca al domo salino sobre el que se encuentra el municipio de Nemocón , Cundinamarca. El objetivo de estudio fue identificar la intrusión del domo debajo de la línea de adquisición.

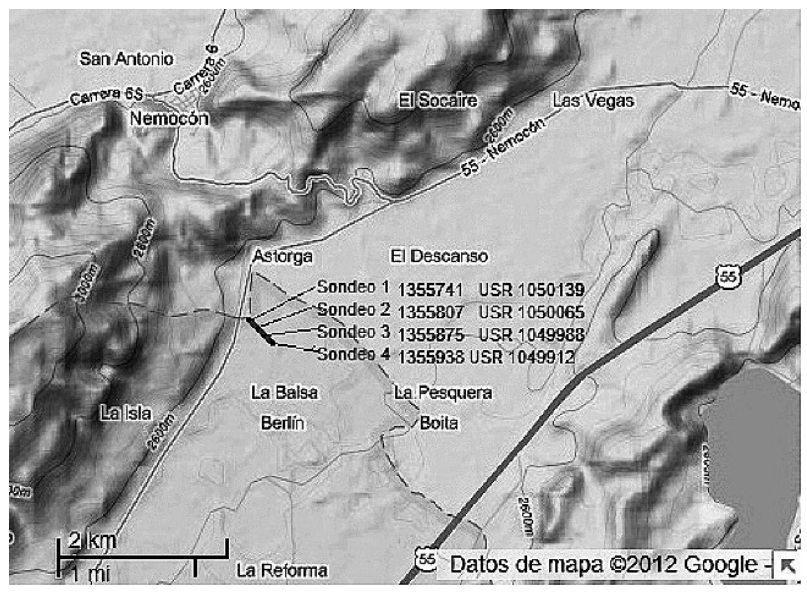

Fig. 10 Ubicación de la línea geográfica de los sondeos realizados. (Fuente: Google maps.)

A continuación se exponen los resultados de campo obtenidos con el equipo de adquisición, esto es, los valores de voltaje en milivoltios y los valores de corriente en miliamperios para cada punto de cada sondeo. Los valores AB/2 y MN/2 de las tablas corresponden a los valores medios de las distancias entre los pares de electrodos $A$ y $\mathrm{B}$, y $\mathrm{M}$ y $\mathrm{N}$, respectivamente. El valor $\mathrm{K}$ corresponde a la constante geométrica del arreglo, y esta depende del tipo de arreglo y de los valores de las distancias electródicas. El valor \a corresponde al valor de la resistividad aparente para el punto que se está midiendo en ese momento.

\section{A. Resultados del primer SEV.}

Los valores de voltaje y corriente obtenidos en campo durante la adquisición del primer SEV se procesaron en el software IPI2Win [5] y los resultados de dicho procesamiento se ilustran en la Fig. 11.

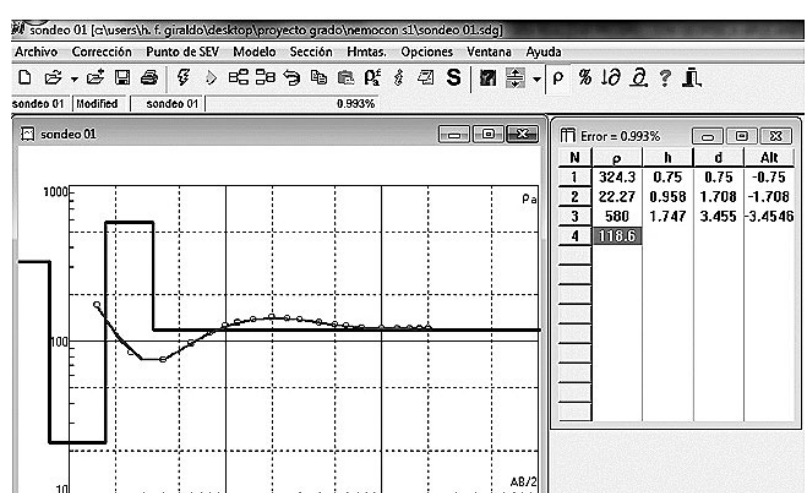

Fig. 11 Inversión de los datos del sondeo uno. 
La gráfica a la izquierda en la Fig. 11 muestra los valores de resistividad aparente sobre la línea roja, y los valores de resistividad real en la gráfica azul. Esta gráfica expone los valores de resistividad respecto de la distancia media entre los electrodos $A$ y $B$.

La tabla de la derecha en la figura muestra los valores de las resistividades (expuestos en azul en la gráfica) y la profundidad de la misma. Esto permite generar un modelo en una dimensión de las resistividades de las capas del subsuelo en profundidad, en el punto central de la línea donde se realizó el sondeo.

Aquí se observa que hay una primera capa con una resistividad de $324.3 \Omega / \mathrm{m}$ y con un espesor de $0.75 \mathrm{~m}$. El modelo también propone una segunda capa con una resistividad de $22.27 \Omega / m$ y con un espesor de $0.96 \mathrm{~m}$. La tercera capa en profundidad expone una resistividad de $580 \Omega / m$ y con un espesor de $1.75 \mathrm{~m}$. Debajo de estas tres capas subyace una cuarta de espesor no definido que posee una resistividad de $118.6 \Omega / \mathrm{m}$. Estos valores están acordes con lo que se espera encontrar en el terreno, que son valores de resistividad correspondientes a calizas y areniscas.

\section{B. Resultados de segundo SEV.}

Los valores de voltaje y corriente obtenidos en campo durante la adquisición del segundo SEV se procesaron en el software IPI2Win y los resultados de dicho procesamiento se ilustran en la Fig. 12.

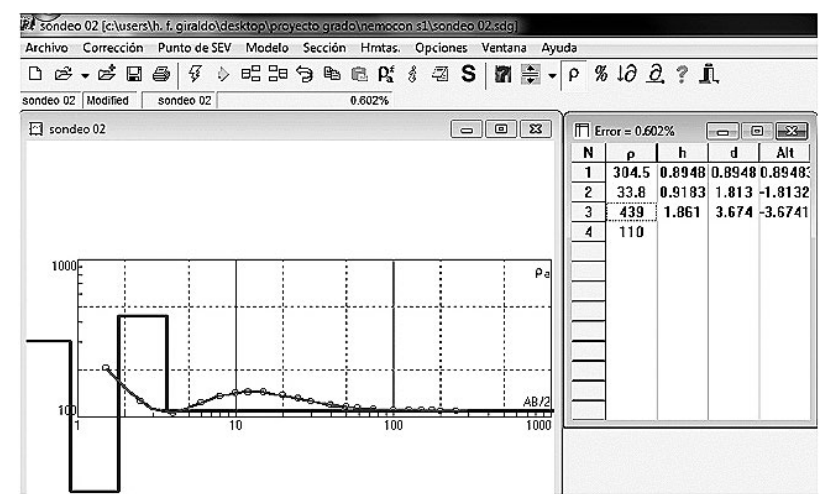

Fig 12. Inversión de datos del segundo sondeo.
La gráfica a la izquierda de la figura 12 muestra los valores de resistividad aparente sobre la línea roja, y los valores de resistividad en la gráfica azul. Esta gráfica expone los valores de resistividad respecto de la distancia media entre los electrodos A y B.

La tabla a la derecha de la figura muestra los valores de las resistividades y la profundidad de las mismas. Esto permite generar un modelo en una dimensión de las resistividades de las capas del subsuelo en profundidad, en el punto donde se realizó el sondeo.

Aquí se observa que hay una primera capa con una resistividad de $304.5 \Omega / m$ y con un espesor de $0.89 \mathrm{~m}$. El modelo también propone una segunda capa con una resistividad de $33.8 \Omega / m$ y con un espesor de $0.92 \mathrm{~m}$. La tercera capa en profundidad expone una resistividad de $439 \Omega / m$ y un espesor de $1.86 \mathrm{~m}$. Debajo de estas tres capas subyace una cuarta de espesor no definido que posee una resistividad de $110 \Omega / \mathrm{m}$. Estos valores están acordes con lo que se espera encontrar en el terreno, que son valores de resistividad correspondientes a calizas y areniscas.

\section{Resultados de tercer SEV.}

Los valores de voltaje y corriente obtenidos en campo durante la adquisición del tercer SEV se procesaron en el software IPI2Win y los resultados de dicho procesamiento se ilustran en la Fig. 13.

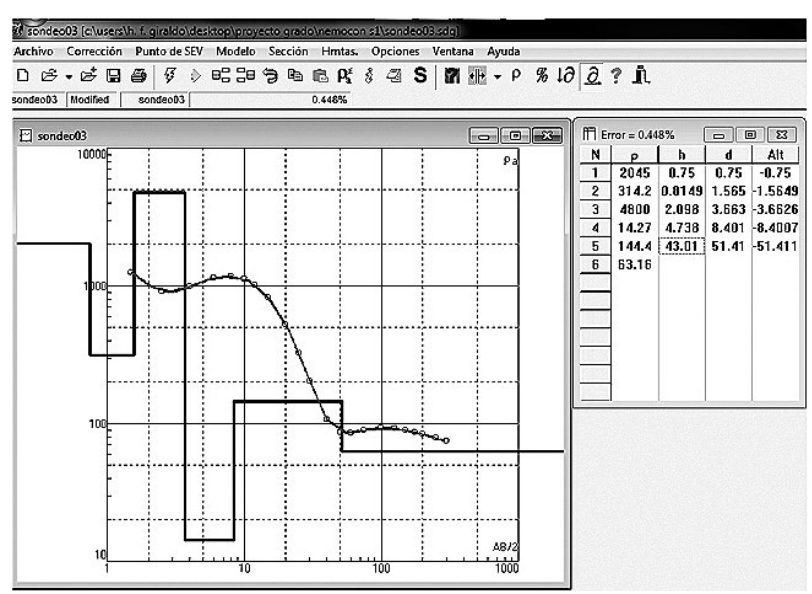

Fig. 13 Inversión de los datos del SEV número tres. 
Para este sondeo se generó un modelo de 6 capas donde la primera capa presenta una resistividad de $2045 \rho / m$ hasta una profundidad de $0.75 \mathrm{~m}$; una segunda capa con una resistividad de 314.2 $\rho / m$ con un espesor de 0.81 metros; una tercera capa con una resistividad de $4800 \rho / \mathrm{m}$ y un espesor de $.09 \mathrm{~m}$, seguida de una capa de $14.27 \rho / \mathrm{m}$ y un espesor de 4,73. La capa siguiente posee una resistividad de $144.4 \mathrm{\rho} / \mathrm{m}$ y un espesor de 43 metros. La capa final exhibe una resistencia de 63.16 $\rho / m$ a una profundidad indeterminada.

\section{Resultado cuarto SEV.}

Los valores de voltaje y corriente obtenidos en campo durante la adquisición del cuarto SEV se procesaron en el software IPI2Win y los resultados de dicho procesamiento se ilustran en la Fig. 14.

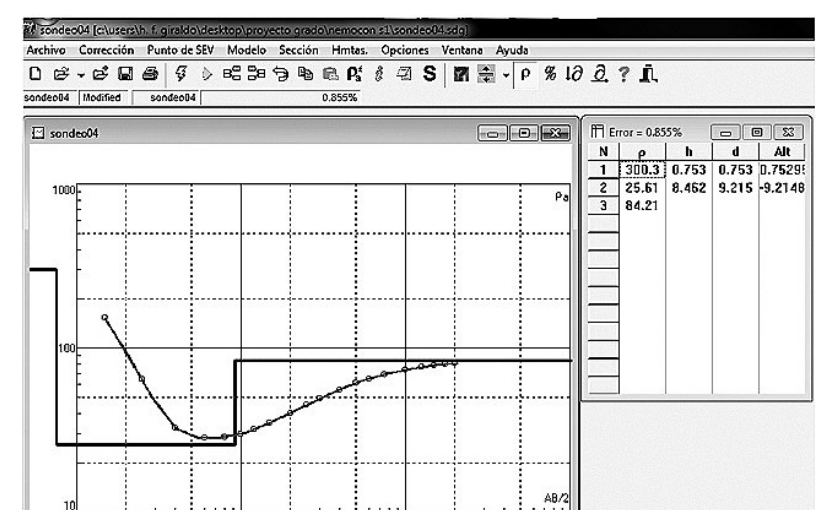

Fig. 14 Inversión de los datos del SEV número cuatro.

La inversión de los datos del cuarto sondeo arrojó un modelo de 3 capas con las siguientes características: una primera capa con una resistividad de $300.1 \rho / m$ que se puede encontrar hasta una profundidad de $0.75 \mathrm{~m}$. Una segunda capa, identificada con una resistividad de $25.61 \mathrm{\rho} / \mathrm{m}$ con un espesor de $8.46 \mathrm{~m}$. Debajo de la segunda capa subyace una tercera capa que presenta una resistividad de $84.21 \mathrm{\rho} / \mathrm{m}$ y posee un espesor indefinido.

\section{F. Perfil geoeléctrico obtenido.}

Los resultados de los cuatro sondeos eléctricos verticales realizados se utilizan en la creación de un pseudo perfil 2D de la línea sobre la cual se realizaron los sondeos. La Fig. 15 muestra el perfil de resistividades aparentes generado. La escala horizontal ubicada en la parte inferior de la figura, indica la distancia en metros entre los sondeos, los cuales se identifican como s1, s2, s3 y s4. La escala vertical, indica la profundidad en metros. En la parte derecha se observa la escala en colores para los valores de resistividad.

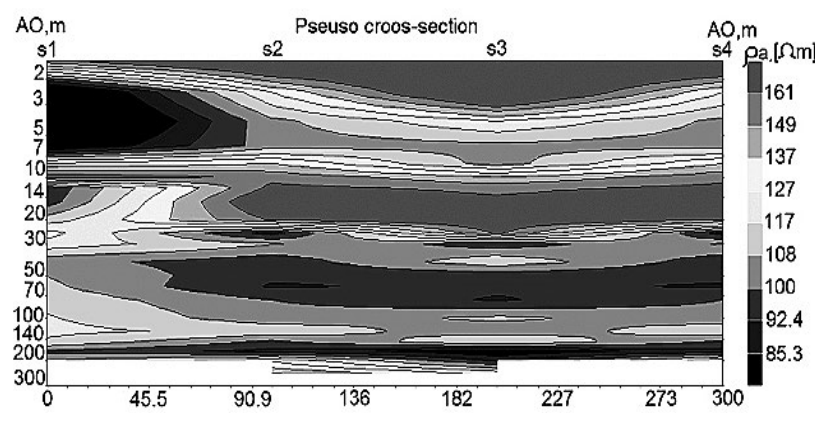

Fig. 15 Perfil geoeléctrico obtenido a partir de los SEV.

\section{Conclusiones}

Este equipo, construido y diseñado para realizar prospección geoeléctrica, demostró estar capacitado para realizar estudios de tomografía geoeléctrica a profundidades de por lo menos 250 metros. Esta profundidad de investigación es dependiente de la longitud de la separación entre los electrodos A y $\mathrm{B}$ y de las condiciones del terreno estudiado.

No se efectúa un análisis matemático profundo y detallado del sistema, debido a su desarrollo eminentemente práctico. Es un equipo basado en una electrónica sencilla, fácil de entender y reparar.

Este es un equipo construido con el objetivo de ser práctico y funcional; por ello, ha sido pensado y realizado con componentes electrónicos de fácil acceso en cualquier mercado local, lo que es un punto a favor en caso de que llegase a sufrir algún tipo de avería, pues retrasar demasiado o suspender totalmente una campaña de adquisición de datos en campo implica costos que no se recuperarían, si no se lograra el objetivo de la misma. 
La portabilidad, resistencia y robustez pensadas en el diseño del equipo para el trabajo en campo abierto, han cumplido con el objetivo deseado cuando se ha requerido.

Se logró obtener un perfil de resistividades de la zona estudiada, y los resultados obtenidos están acordes con lo esperado, según la geología de campo realizada con anterioridad.

\section{Agradecimientos}

Este trabajo fue financiado por la empresa SUBSUELO3D S.A.S, la cual se dedica a fomentar, desarrollar y aplicar la investigación en el campo de la geofísica.

\section{RefERENCIAS}

[1] D.H. Griffiths y R. Favell King "Geofísica aplicada para ingenieros" Paraninfo, p. 231, 1972.

[2] Schlumberger y Wenner.

[3] M. Auge., "Métodos geoeléctricos en la prospección de agua subterránea", p. 30, 2008.

[4] E. Orellana., "Prospección eléctrica en corriente continua," Paraninfo, 2da edición, p. 523, 1982.

[5] M. H. Locke, "Tutorial 2D and 3D electrical image surveys", 2004.

[6] IPI2Win guía de usuario, UNIVERSIDAD ESTATAL DE MOSCÚ, p.33, 2000. 